\title{
Effects of Simultaneous versus Staged VAC Placement in the Treatment of Deep Neck Multiple-Space Infections at a Tertiary Hospital Over a Four-Year Period in China
}

\author{
Weijiao Liu ${ }^{1,2, *}$ \\ Wei Gu ${ }^{1}$,* \\ Xiaofeng Jin ${ }^{1, *}$ \\ Jian Wang ${ }^{1,3}$
}

'Department of Otolaryngology-Head and Neck Surgery, Peking Union Medical College Hospital, Chinese Academy of Medical Sciences and Peking Union Medical College, Beijing, People's Republic of China; ${ }^{2}$ Department of Otolaryngology-Head and Neck Surgery, Beijing Friendship Hospital Pinggu

Campus, Capital Medical University, Beijing, People's Republic of China; ${ }^{3}$ State Key Laboratory of Complex Severe and Rare Diseases, Peking Union Medical College Hospital, Chinese Academy of Medical Sciences and Peking Union Medical College, Beijing, People's Republic of China

*These authors contributed equally to this work
Correspondence: Jian Wang Department of Otolaryngology-Head and Neck Surgery, Peking Union Medical College Hospital, Chinese Academy of Medical Sciences and Peking Union Medical College, No. I Shuaifuyuan, Wangfujing, Dongcheng District, Beijing, 100730, People's Republic of China Email wangjianent@I26.com
Purpose: Surgical drainage is an essential part of treatment for deep neck infections (DNIs) or deep neck multiple-space infections (DNMIs). With the emergence and application of new technologies and new materials, vacuum-assisted closure (VAC) in the treatment of DNMIs has been reported. However, reports on the timing of VAC placement are limited. Herein, we compared simultaneous versus staged VAC placement in the treatment of DNMIs.

Patients and Methods: Medical data from 24 patients with DNMIs who had received VAC treatment in the last five years were analyzed. The patients were classified into a simultaneous VAC placement group (11 patients) and a staged VAC placement group (13 patients) according to the timing of VAC placement when incision and drainage were performed.

Results: No differences in baseline characteristics were found between the two groups. All patients in the two groups survived and recovered. The hospitalization duration (days), time to wound healing (days), number of debridement procedures, and disease course (days) in the simultaneous VAC placement group and staged VAC placement group were 10 (4-18) and 22 (8-35), 21 (4-39) and 50 (9-86), one (1-2) and two (1-4), and 31.5 (11-49) and 56 (19-98), respectively. The results in the simultaneous VAC placement group were better than those in the staged VAC placement group $(\mathrm{P}=0.001$, $0.016,0.045$, and 0.016 , respectively). The numbers of VAC sponge changes in the simultaneous VAC placement group and staged VAC placement group were two (1-2) and two (1-4), respectively, with no statistically significant difference $(\mathrm{P}=0.336)$.

Conclusion: Simultaneous VAC placement during incision and drainage may shorten the wound healing time, hospitalization duration, and disease course and may reduce the number of debridement procedures.

Keywords: deep neck multiple-space infections, DNMIs, necrotizing fasciitis, surgical treatment, negative pressure wound therapy

\section{Introduction}

Deep neck infections (DNIs) are a group of serious bacterial infections occurring in the potential spaces and fascia of the neck. ${ }^{1}$ The incidence of DNIs is not clear. Although many studies have shown that the incidence of DNIs has decreased due to the use of antibiotics and improvements in sanitary conditions, ${ }^{2-4}$ reports also indicate an increased incidence of DNIs in the last 10 years. ${ }^{5,6}$ Deep neck multiple-space infections (DNMIs) are the most serious among all types of DNIs and often spread further to cause mediastinal 
infections, invade the carotid sheath, and possibly compromise the airway, resulting in life-threatening conditions. ${ }^{7,8}$

Treatments for DNMIs include life-supporting measures, surgical drainage, and appropriate use of antibiotics. Among these treatments, surgical drainage is key. ${ }^{9}$ Although traditional extensive surgical drainage is effective for treating DNMIs, some limitations remain for this approach, ie, drainage depends on gravity, the locations for the drainage tube and incision are limited, dressing changes and wound irrigation more than once a day may increase patient suffering, and secondary wound infection may occur. ${ }^{10,11}$ In recent years, vacuum-assisted closure (VAC) in the treatment of DNIs or DNMIs has been reported $^{12-15}$ and has shown positive efficacy, but no reports on the timing of VAC placement are available.

This study aimed to compare the effects of simultaneous VAC placement and staged VAC placement by retrospectively analyzing the medical data of DNMI patients who had received VAC treatment within the last five years.

\section{Patients and Methods}

\section{Patients}

This study was approved by the ethics committee of Peking Union Medical College Hospital (ethics approval No. JS2084). All patients included in the study signed an informed consent form before surgery. The medical data of patients with DNMIs who received VAC treatment at our hospital between January 2015 and December 2019 were collected, and medical records, imaging data, and bacteriological results were reviewed. Patients with DNMIs caused by tumors and trauma were excluded from the study. A total of 24 patients were included.

\section{Surgical Procedures}

Simultaneous VAC placement: All patients underwent general anesthesia following endotracheal intubation or tracheostomy for airway protection before neck incision and drainage. The appropriate incision was made according to the different sites of infection to ensure adequate exposure of the infected area and smooth drainage. The pus was collected during the surgery for bacterial culture and susceptibility testing. After the abscess cavity was exposed, blunt dissection of the infected spaces by fingers was performed for smooth drainage, and the necrotic tissues were removed (Figure 1A). Blunt dissection by fingers is a key step. On the one hand, separating tissues by fingers is safe and has little impact on the complex nerves and vascular tissues of the neck, which is increasingly important when facing unclear anatomical signs in deep neck multiple-space infections. On the other hand, this method is also very effective in opening every potential pus cavity, which promotes the treatment effect. After repeated rinsing of the surgical cavity and verifying that the cervical sheath was free from infections, foam material of appropriate size was placed according to the infected area, and transparent film completely covered the wound to ensure sealing. Then, the VAC device (V.A.C. ${ }^{\circledR}$, Kinetic Concepts Inc, San Antonio Texas, USA) was connected (Figure 1B).

Staged VAC placement: All patients in the staged VAC placement group had received conventional incision and drainage, and the VAC was placed in the same way as that in the simultaneous VAC placement group during repeated debridement (Figure 1C and D).

\section{Evaluation Indicators}

First incision time: the time from DNI onset to the first incision and drainage procedure (the time of puncture was not considered the incision time); hospitalization duration: the time from patient referral to our hospital to patient discharge from our hospital; wound healing time: the time from incision to reaching all four endpoints (self-healing, stitch removal, drainage tube removal, and tracheostomy cannula removal); disease course: the time from DNI onset to complete healing; number of debridement procedures: the number of debridement procedures performed in the operating room (bedside debridement was not included); and number of VAC changes: the number of VAC sponge changes.

\section{Results}

\section{General Information}

All 24 patients who underwent VAC placement were referred to our hospital (a central and tertiary hospital in Northern China) from other hospitals. The results for the simultaneous VAC placement group (11 patients) and staged VAC placement group (13 patients) were as follows: age of the patients: 58 (47-65) and 57 (41-79) years; sex ratio: 9:2 and 11:2; body mass index (BMI): 25.38 (22.09-31.63) and $24.76(17.99-35.16) \mathrm{kg} / \mathrm{m}^{2}$; the proportion of patients with concomitant diabetes: $5 / 11$ (54.5\%) and $7 / 13(53.8 \%)$; the time from onset to incision: seven (5-15) and 10 (4-21) days; and the proportion of patients 


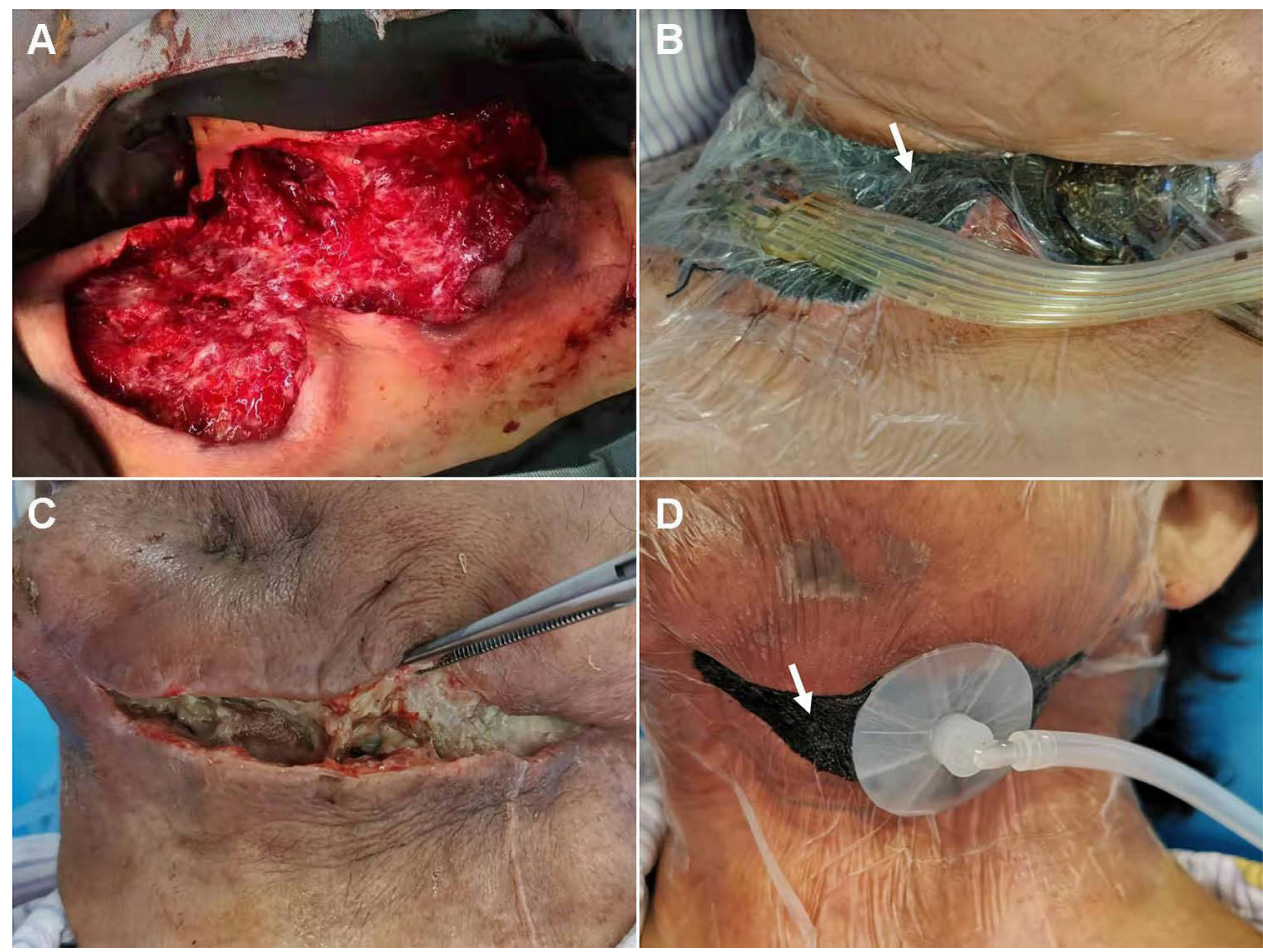

Figure I Vacuum-assisted closure (VAC) in the treatment of deep neck infection. Simultaneous VAC: (A) The abscess cavity was exposed, and necrotic tissues were removed. (B) The foam material was then placed into the infected area, and the transparent film completely covered the wound to ensure sealing. In addition, the VAC device was connected. Staged VAC: (C) Infection wound failed to heal after conventional drainage and repeated debridement. (D) The VAC device was then placed into the infected area to facilitate wound healing. White arrows in (B and D) indicate the foam material and the transparent film, respectively.

undergoing tracheotomy: 5/11 (45.5\%) and 8/13 (61.5\%), respectively. All the differences between the two groups were not statistically significant $(\mathrm{P}=0.586,0.648,0.902$, $0.528,0.301$, and 0.268 , respectively) (Table 1 ).

The ratios of patients with odontogenic infection (patients with a history of toothache and tooth extraction before onset) to patients with nonodontogenic infection in the simultaneous VAC placement group and staged VAC placement group were 6:5 and 6:7, respectively. The imaging and surgery results demonstrated that all the patients had DNMIs. In the simultaneous VAC placement group and the staged VAC placement group, involvement of the submental and submandibular spaces was observed in 9 and 12 patients, parapharyngeal space involvement was observed in
11 and 13 patients, retropharyngeal space involvement was observed in four and eight patients, prevertebral space involvement was observed in one and two patients, and mediastinal infection was observed in four and five patients, respectively. Multiple bacterial infections detected in the initial culture of secretions were found in two patients in the simultaneous VAC placement group and in four patients in the staged VAC placement group (Table 2). The results of initial bacterial culture of secretions from the 24 patients were as follows: Streptococcus anginosus (eight patients), Streptococcus constellatus (five patients), Acinetobacter baumannii (two patients), viridans group streptococci, Staphylococcus epidermidis, Streptococcus parasanguis, coagulase-negative Staphylococcus, and Stenotrophomonas

Table I General Information

\begin{tabular}{|l|l|l|l|}
\hline Patient Demographics & Simultaneous Placement $(\mathbf{n}=\mathbf{I} \mathbf{I})$ & Asynchronous Placement $(\mathbf{n}=\mathbf{I 3})$ & P value \\
\hline Age $($ year) & $58(47-65)$ & $57(4 I-79)$ & 0.586 \\
Male/Female & $9 / 2$ & $1 \mathrm{I} / 2$ & 0.648 \\
$\mathrm{BMI}\left(\mathrm{kg} / \mathrm{m}^{2}\right)$ & $24.76(17.99-35.16)$ & 0.902 \\
Diabetic ratio & $6 / 1 I(54.5 \%)$ & $7 / 13(53.8 \%)$ & 0.528 \\
Incision time after onset (days) & $8(5-16)$ & $10(4-21)$ & $0.30 I$ \\
Tracheotomy & $5 / 1 \mathrm{I}(45.5 \%)$ & $8 / 13(61.5 \%)$ & 0.268 \\
\hline
\end{tabular}


Table 2 Characteristics of Infection

\begin{tabular}{|c|c|c|c|}
\hline & Simultaneous Placement $(n=I I)$ & Asynchronous Placement $(n=\mid 3)$ & P value \\
\hline Odontogenic/Non-odontogenic & $6 / 5$ & $6 / 7$ & 0.608 \\
\hline \multicolumn{4}{|l|}{ Involved Region } \\
\hline Submental and submandibular & 9 & 12 & 0.619 \\
\hline Parapharyngeal & 11 & 13 & - \\
\hline Retropharyngeal & 4 & 8 & 0.472 \\
\hline Prevertebral & 1 & 2 & 0.684 \\
\hline Mediastinal infection & 4 & 5 & 0.443 \\
\hline Multiple bacterial infections & 2 & 4 & 0.344 \\
\hline Negative of bacterial culture & 3 & 2 & 0.262 \\
\hline
\end{tabular}

maltophilia (one patient each). No pathogenic bacteria were found in the secretion cultures of eight patients.

\section{Clinical Outcomes}

The treatment for all 24 patients was successful, and no deaths occurred. The hospitalization duration (days), wound healing time (days), number of debridement procedures, and disease course (days) in the simultaneous VAC placement group and staged VAC placement group were 10 (4-18) and 22 (8-35), 21 (4-39) and 50 (9-86), one (12) and two (1-4), and $31.5(11-49)$ and 56 (19-98), respectively. All the results in the simultaneous VAC placement group were better than those in the staged VAC placement group $(\mathrm{P}=0.001,0.016,0.045$, and 0.016, respectively). The numbers of VAC sponge changes in the simultaneous VAC placement group and the staged VAC placement group were two (1-2) and two (1-4), respectively, and the difference between the two groups was not statistically significant $(\mathrm{P}=0.336)$ (Table 3$)$.

\section{Discussion}

Sufficient surgical drainage and clearance of necrotic tissues are particularly important in the treatment of DNIs or DNMIs. ${ }^{9}$ An extensive incision is necessary for the traditional approach, which depends on gravity for drainage and requires repeated dressing changes and debridement. The traditional approach is effective, but patients suffer from repeated dressing changes and debridement, infections require a long time for resolution, and the likelihood of nosocomial infection increases. ${ }^{16}$

Kostiuchenok (1986) first reported the use of VAC in the treatment of 116 patients with pyogenic infections in Russia and found that wound healing was promoted. ${ }^{17}$ Since then, VAC treatment has become popular for use in wound healing. Schuster et $\mathrm{al}^{14}$ and Gallo et al ${ }^{18}$ first reported the use of VAC in the treatment of facial infections and DNIs or DNMIs, and good efficacy was achieved in both studies. VAC is widely believed to promote wound healing and reduce infections by producing local negative pressure to absorb wound secretions and blood, thus promoting reparative granulation tissue formation. ${ }^{19,20}$ Balci et $^{2}{ }^{21}$ and Chen et $\mathrm{al}^{12}$ reported 11 and seven cases of VAC in the treatment of necrotizing fasciitis of the head and neck, respectively. Cao et $\mathrm{al}^{11}$ reported seven cases of VAC in the treatment of serious deep neck and face infections. These three studies concluded that VAC had positive efficacy in the treatment of DNIs or DNMIs according to the descriptive analysis of the included cases. Unfortunately, these articles did not

Table 3 Evaluation of Efficacy

\begin{tabular}{|l|l|l|l|}
\hline & Simultaneous Placement $(\mathbf{n}=\mathbf{I I})$ & Asynchronous Placement $(\mathbf{n}=\mathbf{I 3})$ & P value \\
\hline Hospital stays length (days) & $10(4-18)$ & $22(8-35)$ & $0.00 I^{* *}$ \\
\hline Healing length (days) & $2 I(4-39)$ & $50(9-86)$ & $0.016^{*}$ \\
$\quad$ Number of debridement & I (I-2) & $2(I-4)$ & $0.045^{*}$ \\
$\quad$ Number of replacement & $2(I-2)$ & $2(I-4)$ & 0.336 \\
\hline Disease course (days) & $3 I .5(I I-49)$ & $56(19-98)$ & $0.016^{*}$ \\
\hline
\end{tabular}

Notes: *Statistical significance; **Significant statistical significance. 
report whether differences in the timing of VAC placement cause differences in efficacy.

This study reported the use of VAC in the treatment of 24 patients with DNMIs, representing the largest number of enrolled patients undergoing VAC placement for DNI or DNMI treatment among currently available articles. This retrospective analysis showed that baseline disease characteristics between the two groups were comparable. The findings of this study demonstrated that the hospitalization duration, wound healing time, number of debridement procedures, and disease course in the simultaneous VAC placement group were significantly better than those in the staged VAC placement group. However, previous studies did not report the impact of VAC placement timing on efficacy. The meta-analyses performed by Sahebally et $\mathrm{al}^{22}$ Hyldig et $\mathrm{al}^{23} \mathrm{Wu}$ et $\mathrm{al}^{24}$ and Gabriel et $\mathrm{al}^{25}$ were reviewed, and the results showed that simultaneous VAC placement during wound closure reduced the likelihood of surgical site infections, including those resulting from abdominal surgery, thoracic surgery, and breast surgery. A meta-analysis by Liu et $\mathrm{al}^{26}$ showed that in patients with infectious wounds such as diabetic foot ulcers, VAC can increase the proportion of diabetes patients with postoperative healing of foot wounds and foot ulcers and shorten the healing time compared with a traditional nonnegative pressure wound therapy dressing. These findings indicate that early VAC placement can reduce the incidence of wound (a clean wound or a contaminated wound) infection and promote wound healing. In theory, the mechanism of VAC in reducing infections and promoting wound healing involves improving the blood flow around wounds, reducing tissue edema, and stimulating the formation of reparative granulation tissue. ${ }^{19,20,27}$ However, whether VAC can reduce bacterial loads remains debatable. ${ }^{27-29}$ These findings can explain why DNMI patients who received simultaneous VAC placement had shorter healing times.

This study has the following limitations: this was a retrospective nonrandomized controlled study, and the sample size was not sufficiently large. Furthermore, in all patients who underwent VAC placement, the carotid sheath was found to be intact and free from infection during surgery; concomitant carotid sheath infection is one cause of fatal DNIs. Finally, the patients included in this study does not represent all patients with DNIs, especially those with fatal massive bleeding.

\section{Conclusion}

Although our study has some limitations, the findings demonstrate that simultaneous VAC placement may shorten the wound healing time, hospitalization duration, and disease course and may reduce the number of debridement procedures compared with staged VAC placement.

\section{Acknowledgments}

This study was funded by the Nature Science Foundation of Beijing, China (Grant No. 7192171).

\section{Disclosure}

The authors report no conflicts of interest in this work.

\section{References}

1. Osborn TM, Assael LA, Bell RB. Deep space neck infection: principles of surgical management. Oral Maxillofac Surg Clin North Am. 2008;20(3):353-365. doi:10.1016/j.coms.2008.04.002

2. Wang L-F, Tai C-F, Kuo W-R, Chien C-Y. Predisposing factors of complicated deep neck infections: 12-year experience at a single institution. J Otolaryngol Head Neck Surg. 2010;39(4):335-341.

3. Celakovsky P, Kalfert D, Tucek L, et al. Deep neck infections: risk factors for mediastinal extension. Eur Arch Otorhinolaryngol. 2014;271(6):1679-1683. doi:10.1007/s00405-013-2651-5

4. Buckley J, Harris AS, Addams-Williams J. Ten years of deep neck space abscesses. J Laryngol Otol. 2019;133(4):324-328. doi:10.1017/ S0022215119000458

5. Lau AS, Upile NS, Wilkie MD, Leong SC, Swift AC. The rising rate of admissions for tonsillitis and neck space abscesses in England, 1991-2011. Ann R Coll Surg Engl. 2014;96(4):307-310. doi:10.1308/ $003588414 X 13946184900363$

6. Seppänen L, Rautemaa R, Lindqvist C, Lauhio A. Changing clinical features of odontogenic maxillofacial infections. Clin Oral Investig. 2010;14(4):459-465. doi:10.1007/s00784-009-0281-5

7. Levitt GW. Cervical fascia and deep neck infections. Laryngoscope. 1970;80(3):409-435. doi:10.1288/00005537-197003000-00004

8. Saito Y, Asami M, Miki A, et al. Deep neck infection complicated by phlegmonous esophagitis and mediastinitis. Ann Thorac Surg. 2021;111(6):e403-e406. doi:10.1016/j.athoracsur.2020.08.101

9. Flynn TR, Shanti RM, Levi MH, Adamo AK, Kraut RA, Trieger N. Severe odontogenic infections, part 1: prospective report. J Oral Maxillofac Surg. 2006;64(7):1093-1103. doi:10.1016/j.joms.20 06.03.015

10. Lawrence R, Bateman N. Controversies in the management of deep neck space infection in children: an evidence-based review. Clin Otolaryngol. 2017;42(1):156-163. doi:10.1111/coa.12692

11. Cao J, Liu Z, Ma D, Shen S, Wang X. Modified usage of negative pressure wound therapy for the management of severe deep fascial space infections in the head and neck. Infect Drug Resist. 2020;13:781-788. doi:10.2147/IDR.S243794

12. Chen S-J, Chen Y-X, Xiao J-R, Wei X-Z, Chen S-M, Jiang W-Z. Negative pressure wound therapy in necrotizing fasciitis of the head and neck. J Oral Maxillofac Surg. 2019;77(1):87-92. doi:10.1016/j. joms.2018.08.016

13. Novelli G, Catanzaro S, Canzi G, Sozzi D, Bozzetti A. Vacuum assisted closure therapy in the management of cervico-facial necrotizing fasciitis: a case report and review of the literature. Minerva Stomatol. 2014;63(4):135-144. 
14. Schuster R, Moradzadeh A, Waxman K. The use of vacuum-assisted closure therapy for the treatment of a large infected facial wound. Am Surg. 2006;72(2):129-131. doi:10.1177/000313480607200206

15. Gu X, Chen W, Yuan K, Tan J, Sun S. The efficacy of artificial dermis combined with continuous vacuum sealing drainage in deep neck multiple spaces infection treatment. Medicine. 2021;100(5): e24367. doi:10.1097/MD.0000000000024367

16. Dellinger EP. Prevention of hospital-acquired infections. Surg Infect (Larchmt). 2016;17(4):422-426. doi:10.1089/sur.2016.048

17. Kostiuchenok BM, Kolker II, Karlov VA, Ignatenko SN, Muzykant LI. [Vacuum treatment in the surgical management of suppurative wounds]. Vestn Khir Im I I Grek. 1986;137(9):18-21. Russian.

18. Gallo O, Deganello A, Meccariello G, Spina R, Peris A. Vacuumassisted closure for managing neck abscesses involving the mediastinum. Laryngoscope. 2012;122(4):785-788. doi:10.1002/ lary. 22403

19. Costa ML, Achten J, Bruce J, et al. Effect of negative pressure wound therapy vs standard wound management on 12-month disability among adults with severe open fracture of the lower limb: the WOLLF randomized clinical trial. JAMA. 2018;319(22):2280-2288. doi:10.1001/jama.2018.6452

20. Pollak AN, Jones AL, Castillo RC, Bosse MJ, MacKenzie EJ; LEAP Study Group. The relationship between time to surgical debridement and incidence of infection after open high-energy lower extremity trauma. J Bone Joint Surg Am. 2010;92(1):7-15. doi:10.2106/JBJS. H.00984

21. Balcı MK, Ciğer E, Arslanoğlu S, Işlek A. Necrotizing fasciitis of the head and neck: our experience with vacuum-assisted closure therapy. Eur Arch Otorhinolaryngol. 2018;275(10):2555-2562. doi:10.1007/ s00405-018-5096-z
22. Sahebally SM, McKevitt K, Stephens I, et al. Negative pressure wound therapy for closed laparotomy incisions in general and colorectal surgery: a systematic review and meta-analysis. JAMA Surg. 2018;153(11):e183467. doi:10.1001/jamasurg.2018.3467

23. Hyldig N, Birke-Sorensen H, Kruse M, et al. Meta-analysis of negative-pressure wound therapy for closed surgical incisions. $\mathrm{Br}$ J Surg. 2016;103(5):477-486. doi:10.1002/bjs.10084

24. Wu RT, Sumpio BJ, Miller S, Sumpio BE. Use of closed-incision negative-pressure therapy: cardiothoracic and vascular surgery. Plast Reconstr Surg. 2019;143(1S):31S-35S. doi:10.1097/PRS.0000 000000005310

25. Gabriel A, Sigalove S, Sigalove N, et al. The impact of closed incision negative pressure therapy on postoperative breast reconstruction outcomes. Plast Reconstr Surg Glob Open. 2018;6(8):e1880. doi:10.1097/GOX.0000000000001880

26. Liu Z, Dumville JC, Hinchliffe RJ, et al. Negative pressure wound therapy for treating foot wounds in people with diabetes mellitus. Cochrane Database Syst Rev. 2018;10:CD010318. doi:10.1002/ 14651858.CD010318.pub3

27. Malmsjö M, Ingemansson R, Martin R, Huddleston E. Wound edge microvascular blood flow: effects of negative pressure wound therapy using gauze or polyurethane foam. Ann Plast Surg. 2009;63 (6):676-681. doi:10.1097/SAP.0b013e31819ae01b

28. Braakenburg A, Obdeijn MC, Feitz R, van Rooij IALM, van Griethuysen AJ, Klinkenbijl JHG. The clinical efficacy and cost effectiveness of the vacuum-assisted closure technique in the management of acute and chronic wounds: a randomized controlled trial. Plast Reconstr Surg. 2006;118(2):390-397; discussion 398-400. doi:10.1097/01.prs.0000227675.63744.af

29. Mouës CM, Heule F, Hovius SER. A review of topical negative pressure therapy in wound healing: sufficient evidence? Am J Surg. 2011;201(4):544-556. doi:10.1016/j.amjsurg.2010.04.029
Infection and Drug Resistance

\section{Publish your work in this journal}

Infection and Drug Resistance is an international, peer-reviewed openaccess journal that focuses on the optimal treatment of infection (bacterial, fungal and viral) and the development and institution of preventive strategies to minimize the development and spread of resistance. The journal is specifically concerned with the epidemiology of antibiotic resistance and the mechanisms of resistance development and diffusion in both hospitals and the community. The manuscript management system is completely online and includes a very quick and fair peerreview system, which is all easy to use. Visit http://www.dovepress.com/ testimonials.php to read real quotes from published authors. 